\title{
FOCK FACTORIZATION OF B-VALUED ANALYTIC MAPPINGS ON A HILBERT INDUCTIVE LIMIT
}

\author{
CAISHI WANG ${ }^{\otimes}$, YULAN ZHOU, DECHENG FENG and QI HAN
}

(Received 9 May 2009)

\begin{abstract}
Let $\mathcal{N}^{*}$ be a Hilbert inductive limit and $X$ a Banach space. In this paper, we obtain a necessary and sufficient condition for an analytic mapping $\Psi: \mathcal{N}^{*} \mapsto X$ to have a factorization of the form $\Psi=T \circ \mathcal{E}$, where $\mathcal{E}$ is the exponential mapping on $\mathcal{N}^{*}$ and $T: \Gamma\left(\mathcal{N}^{*}\right) \mapsto X$ is a continuous linear operator, where $\Gamma\left(\mathcal{N}^{*}\right)$ denotes the Boson Fock space over $\mathcal{N}^{*}$. To prove this result, we establish some kernel theorems for multilinear mappings defined on multifold Cartesian products of a Hilbert space and valued in a Banach space, which are of interest in their own right. We also apply the above factorization result to white noise theory and get a characterization theorem for white noise testing functionals.
\end{abstract}

2000 Mathematics subject classification: primary 60H40; secondary 46G20.

Keywords and phrases: inductive limit, B-valued analytic mapping, Fock factorization, white noise.

\section{Introduction}

By a Hilbert inductive limit we mean the inductive limit of a family of Hilbert spaces satisfying certain conditions, which belongs to the category of local convex topological vector spaces. Hilbert inductive limits play a crucial role in white noise theory. For example, the domain of white noise testing functionals is exactly a Hilbert inductive limit, and the space of white noise generalized functionals also forms a Hilbert inductive limit. White noise theory is essentially an infinite-dimensional analogue of Schwartz generalized function theory, which was initiated by Hida in 1975 and has now been considerably developed and successfully applied to many research fields, including stochastic analysis and quantum physics (see, for example, [1-4]).

Let $\mathcal{N}^{*}$ be a Hilbert inductive limit and $X$ a Banach space. In this paper we are interested in analytic mappings defined on $\mathcal{N}^{*}$ and valued in $X$. More precisely, we attempt to find necessary and sufficient conditions for an analytic mapping $\Psi: \mathcal{N}^{*} \mapsto X$ to have a factorization of the form $\Psi=T \circ \mathcal{E}$, where $\mathcal{E}$ is the exponential mapping on $\mathcal{N}^{*}$ and $T: \Gamma\left(\mathcal{N}^{*}\right) \mapsto X$ is a continuous linear operator, where $\Gamma\left(\mathcal{N}^{*}\right)$ is the Boson Fock space over $\mathcal{N}^{*}$ (see Section 3 for the definition).

Supported by National Natural Science Foundation of China (10571065), Natural Science Foundation of Gansu Province (0710RJZA106) and NWNU-KJCXGC, PR China.

(c) 2009 Australian Mathematical Publishing Association Inc. 0004-9727/2009 \$16.00 
Our motivation for this study comes from a problem in white noise theory. Let $(E) \subset\left(L^{2}\right) \subset(E)^{*}$ be the classical white noise analysis framework over a real Gel'fand triple $E \subset \mathcal{H} \subset E^{*}$. Elements of $(E)$ are known as white noise testing functionals, while elements of $(E)^{*}$ are known as white noise generalized functionals (see, for example, $[2,3]$ ). It is known that the complexification $E_{c}^{*}$ of $E^{*}$ is a Hilbert inductive limit. Through the Wiener-Itô-Segal isomorphism, we can identify $(E)^{*}$ with $\Gamma\left(E_{c}^{*}\right)$, the Boson Fock space over $E_{c}^{*}$. Hence each $\varphi \in(E)$ can be viewed as a $\mathbb{C}$-valued continuous linear functional on $\Gamma\left(E_{c}^{*}\right)$ and the composition $\varphi \circ \mathcal{E}$ then makes sense as a $\mathbb{C}$-valued functional on $E_{c}^{*}$, where $\mathcal{E}$ is the exponential mapping on $E_{c}^{*}$. One natural problem is to find necessary and sufficient conditions for a functional $G: E_{c}^{*} \mapsto \mathbb{C}$ to have a factorization of the form $G=\varphi \circ \mathcal{E}$, with $\varphi$ being a certain white noise testing functional. This problem is clearly a very special case of what we study in the present paper.

The paper is organized as follows. In Section 2 we establish some kernel theorems for multilinear mappings defined on multifold Cartesian products of a Hilbert space and valued in a Banach space, which will be used to prove our main results. In Section 3, we state and prove our main results. We first introduce notions and notation concerning a Hilbert inductive limit. Then we prove our main theorems, which provide a necessary and sufficient condition for an analytic mapping $\Psi: \mathcal{N}^{*} \mapsto X$ to have a factorization of the form $\Psi=T \circ \mathcal{E}$, with $T: \Gamma\left(\mathcal{N}^{*}\right) \mapsto X$ being a continuous linear operator. In the last section, we apply our main results to white noise theory and get a characterization theorem for white noise testing functionals.

\section{Kernel theorems for B-valued multilinear mappings}

In this section we prove some kernel theorems for multilinear mappings defined on multifold Cartesian products of a Hilbert space and valued in a Banach space. These kernel theorems will be used to prove our main theorems.

Throughout this section, $\mathbb{K}$ denotes either the real numbers $\mathbb{R}$ or the complex numbers $\mathbb{C}$.

Let $H$ be a separable Hilbert space over $\mathbb{K}$ with inner product $\langle\cdot, \cdot\rangle$ and norm $|\cdot|$. Let $X$ be a Banach space over $\mathbb{K}$ with norm $\|\cdot\|_{X}$. We denote by $\langle\cdot, \cdot\rangle_{X^{*} \times X}$ the canonical bilinear form on $X^{*} \times X$, where $X^{*}$ is the dual of $X$.

Let $n \geq 1$ and $M: H^{n} \mapsto X$ an $n$-linear mapping. $M$ is called bounded if $\|M\|<$ $\infty$, where $\|M\|$ is defined by

$$
\begin{gathered}
\|M\|=\sup \left\{\left\|M\left(h_{1}, h_{2}, \ldots, h_{n}\right)\right\|_{X}|| h_{1}|\leq 1,| h_{2}|\leq 1, \ldots,| h_{n} \mid \leq 1,\right. \\
\left.\left(h_{1}, h_{2}, \ldots, h_{n}\right) \in H^{n}\right\} .
\end{gathered}
$$

In that case, $\|M\|$ is called the norm of $M$.

Definition 2.1. Let $n \geq 1$. A bounded $n$-linear mapping $M: H^{n} \mapsto X$ is said to be strongly bounded if there exists an orthonormal basis $\left\{e_{k}\right\}_{k \geq 1}$ of $H$ such that

$$
\|M\|_{s}^{2} \equiv \sup _{\|g\|=1, g \in X^{*}} \sum_{j_{1}, j_{2}, \ldots, j_{n}}\left|\left\langle g, M\left(e_{j_{1}}, e_{j_{2}}, \ldots, e_{j_{n}}\right)\right\rangle_{X^{*} \times X}\right|^{2}<\infty
$$

where $\sum_{j_{1}, j_{2}, \ldots, j_{n}} \equiv \sum_{j_{1}, j_{2}, \ldots, j_{n}=1}^{\infty}$. In that case, $\|M\|_{s}$ is called the strong norm of $M$. 
As is shown below, $\|M\|_{s}$ is actually independent of the choice of the orthonormal basis $\left\{e_{k}\right\}_{k \geq 1}$.

Let $H^{\otimes n}$ be the $n$-fold Hilbert tensor product of $H$. By convention, the inner product and norm of $H^{\otimes n}$ are still denoted by $\langle\cdot, \cdot\rangle$ and $|\cdot|$, respectively.

THEOREM 2.2. Let $n \geq 1$. If $M: H^{n} \mapsto X$ is a strongly bounded $n$-linear mapping, then there exists a unique bounded linear operator $T_{M}: H^{\otimes n} \mapsto X$ such that

$$
M\left(h_{1}, h_{2}, \ldots, h_{n}\right)=T_{M}\left(h_{1} \otimes h_{2} \otimes \cdots \otimes h_{n}\right), \quad\left(h_{1}, h_{2}, \ldots, h_{n}\right) \in H^{n},
$$

and moreover, $\left\|T_{M}\right\|=\|M\|_{s}$, where $\left\|T_{M}\right\|$ stands for the usual operator norm.

Proof. Obviously, $T_{M}$ is unique if it exists. To prove the existence, we define a mapping $M_{+}: X^{*} \mapsto\left(H^{\otimes n}\right)^{*}$ as

$$
M_{+} g=\sum_{j_{1}, j_{2}, \ldots, j_{n}}\left\langle g, M\left(e_{j_{1}}, e_{j_{2}}, \ldots, e_{j_{n}}\right)\right\rangle_{X^{*} \times X} R\left(e_{j_{1}} \otimes e_{j_{2}} \otimes \cdots \otimes e_{j_{n}}\right), \quad g \in X^{*}
$$

where $\left\{e_{k}\right\}_{k \geq 1}$ is an orthonormal basis of $H$ and $R: H^{\otimes n} \mapsto\left(H^{\otimes n}\right)^{*}$ is the Riesz mapping. It can be easily verified that $M_{+}: X^{*} \mapsto\left(H^{\otimes n}\right)^{*}$ is a bounded linear operator and

$$
\left\|M_{+} g\right\|_{\left(H^{\otimes n}\right)^{*}}^{2}=\sum_{j_{1}, j_{2}, \ldots, j_{n}}\left|\left\langle g, M\left(e_{j_{1}}, e_{j_{2}}, \ldots, e_{j_{n}}\right)\right\rangle_{X^{*} \times X}\right|^{2}, \quad g \in X^{*},
$$

which means $\left\|M_{+}\right\|=\|M\|_{s}$.

For $\left(h_{1}, h_{2}, \ldots, h_{n}\right) \in H^{n}$ and $g \in X^{*}$, it follows that

$$
\begin{aligned}
\left\langle M_{+}\right. & \left.g, h_{1} \otimes h_{2} \otimes \cdots \otimes h_{n}\right\rangle_{\left(H^{\otimes n}\right)^{*} \times H^{\otimes n}} \\
& =\sum_{j_{1}, j_{2}, \ldots, j_{n}}\left\langle g, M\left(e_{j_{1}}, e_{j_{2}}, \ldots, e_{j_{n}}\right)\right\rangle_{X^{*} \times X}\left\langle h_{1}, e_{j_{1}}\right\rangle\left\langle h_{2}, e_{j_{2}}\right\rangle \cdots\left\langle h_{n}, e_{j_{n}}\right\rangle \\
& =\sum_{j_{1}, j_{2}, \ldots, j_{n}}\left\langle g, M\left(\left\langle h_{1}, e_{j_{1}}\right\rangle e_{j_{1}},\left\langle h_{2}, e_{j_{2}}\right\rangle e_{j_{2}}, \ldots,\left\langle h_{n}, e_{j_{n}}\right\rangle e_{j_{n}}\right)\right\rangle_{X^{*} \times X} \\
& =\left\langle g, M\left(h_{1}, h_{2}, \ldots, h_{n}\right)\right\rangle_{X^{*} \times X} .
\end{aligned}
$$

Now let $J_{1}: H^{\otimes n} \mapsto\left(H^{\otimes n}\right)^{* *}$ and $J_{2}: X \mapsto X^{* *}$ be the natural embedding mappings and denote by $M_{+}^{*}$ the adjoint of $M_{+}$. Then, for $\left(h_{1}, h_{2}, \ldots, h_{n}\right) \in H^{n}$ and $g \in X^{*}$,

$$
\begin{aligned}
\left\langle M_{+}^{*}\right. & \left.J_{1}\left(h_{1} \otimes h_{2} \otimes \cdots \otimes h_{n}\right), g\right\rangle_{X^{* *} \times X^{*}} \\
& =\left\langle J_{1}\left(h_{1} \otimes h_{2} \otimes \cdots \otimes h_{n}\right), M_{+} g\right\rangle_{\left(H^{\otimes n}\right)^{* *} \times\left(H^{\otimes n}\right)^{*}} \\
& =\left\langle M_{+} g, h_{1} \otimes h_{2} \otimes \cdots \otimes h_{n}\right\rangle_{\left(H^{\otimes n}\right)^{*} \times H^{\otimes n}} \\
& =\left\langle g, M\left(h_{1}, h_{2}, \ldots, h_{n}\right)\right\rangle_{X^{*} \times X} \\
& =\left\langle J_{2} M\left(h_{1}, h_{2}, \ldots, h_{n}\right), g\right\rangle_{X^{* *} \times X^{*}},
\end{aligned}
$$

which implies that, for each $\left(h_{1}, h_{2}, \ldots, h_{n}\right) \in H^{n}$,

$$
M_{+}^{*} J_{1}\left(h_{1} \otimes h_{2} \otimes \cdots \otimes h_{n}\right)=J_{2} M\left(h_{1}, h_{2}, \ldots, h_{n}\right) \in J_{2}(X) .
$$


Since $\left\{h_{1} \otimes h_{2} \otimes \cdots \otimes h_{n} \mid\left(h_{1}, h_{2}, \ldots, h_{n}\right) \in H^{n}\right\}$ is total in $H^{\otimes n}$ and $J_{2}(X)$ is a closed subspace of $X^{* *}$, it follows that

$$
M_{+}^{*} J_{1}\left(H^{\otimes n}\right) \subset J_{2}(X)
$$

Hence $T_{M} \equiv J_{2}^{-1} M_{+}^{*} J_{1}$ is a bounded linear operator from $H^{\otimes n}$ to $X$. It follows from (2.5) that

$$
M\left(h_{1}, h_{2}, \ldots, h_{n}\right)=T_{M}\left(h_{1} \otimes h_{2} \otimes \cdots \otimes h_{n}\right), \quad\left(h_{1}, h_{2}, \ldots, h_{n}\right) \in H^{n} .
$$

Finally,

$$
\begin{aligned}
\left\|T_{M}\right\| & =\sup _{|u|=1, u \in H^{\otimes n}}\left\|T_{M} u\right\|_{X}=\sup _{|u|=1, u \in H^{\otimes n}}\left\|J_{2}^{-1} M_{+}^{*} J_{1} u\right\|_{X} \\
& =\sup _{|u|=1, u \in H^{\otimes n}}\left\|M_{+}^{*} J_{1} u\right\|_{X^{* *}} \\
& =\sup _{\|v\|=1, v \in\left(H^{\otimes n}\right)^{* *}}\left\|M_{+}^{*} v\right\|_{X^{* *}}=\left\|M_{+}^{*}\right\|=\left\|M_{+}\right\|=\|M\|_{s} .
\end{aligned}
$$

This completes the proof.

REMARK 2.3. According to Theorem 2.2, if $M: H^{n} \mapsto X$ is a strongly bounded $n$ linear mapping, then $\|M\| \leq\|M\|_{s}$.

Let $H^{\widehat{\otimes} n}$ be the $n$-fold symmetric Hilbert tensor product of $H$, which is a closed subspace of $H^{\otimes n}$. Note that $H^{\widehat{\otimes} 0}=\mathbb{K}$. By convention, $H^{\widehat{\otimes} n}$ is endowed with the inner product $n !\langle\cdot, \cdot\rangle$ instead, which is equivalent to the inner product $\langle\cdot, \cdot\rangle$ of $H^{\otimes n}$. Hence $\|\cdot\|_{H^{\widehat{\otimes}}}=\sqrt{n !} \cdot \cdot \mid$.

THEOREM 2.4. Let $n \geq 1$. If $M: H^{n} \mapsto X$ is a strongly bounded symmetric $n$-linear mapping, then there exists a unique bounded linear operator $L_{M}: H^{\widehat{\otimes} n} \mapsto X$ such that

$$
M\left(h_{1}, h_{2}, \ldots, h_{n}\right)=L_{M}\left(h_{1} \widehat{\otimes} h_{2} \widehat{\otimes} \ldots \widehat{\otimes} h_{n}\right), \quad\left(h_{1}, h_{2}, \ldots, h_{n}\right) \in H^{n},
$$

and moreover,

$$
\left\|L_{M}\right\|=\frac{1}{\sqrt{n !}}\|M\|_{s} .
$$

PRoOF. By Theorem 2.2, there is a unique bounded linear operator $T_{M}: H^{\otimes n} \mapsto X$ such that

$$
M\left(h_{1}, h_{2}, \ldots, h_{n}\right)=T_{M}\left(h_{1} \otimes h_{2} \otimes \cdots \otimes h_{n}\right), \quad\left(h_{1}, h_{2}, \ldots, h_{n}\right) \in H^{n} .
$$

Put $L_{M}=\left.T_{M}\right|_{H^{\widehat{\otimes} n}}$. Then it is easy to verify that $L_{M}: H^{\widehat{\otimes} n} \mapsto X$ is a bounded linear operator and, moreover, $L_{M}$ satisfies equality (2.6). 
Taking an orthonormal basis $\left\{e_{k}\right\}_{k \geq 1}$ of $H$, we have

$$
\|M\|_{s}^{2}=\sup _{\|g\|=1, g \in X^{*}} \sum_{j_{1}, j_{2}, \ldots, j_{n}}\left|\left\langle g, M\left(e_{j_{1}}, e_{j_{2}}, \ldots, e_{j_{n}}\right)\right\rangle_{X^{*} \times X}\right|^{2} .
$$

It is known that

$$
\left\{\frac{e_{i_{1}}^{\otimes r_{1}} \widehat{\otimes} e_{i_{2}}^{\otimes r_{2}} \widehat{\otimes} \cdots \widehat{\otimes} e_{i_{k}}^{\otimes r_{k}}}{\sqrt{r_{1} ! r_{2} ! \cdots r_{k} !}} \mid 1 \leq i_{1}<i_{2}<\cdots<i_{k}, r_{1}+r_{2}+\cdots+r_{k}=n, 1 \leq k \leq n\right\},
$$

where $1 \leq r_{1}, r_{2}, \ldots, r_{k} \leq n$, constitutes an orthonormal basis of $H^{\widehat{\otimes} n}$. Hence

$$
\begin{aligned}
\left\|L_{M}\right\|^{2} & =\left\|L_{M}^{*}\right\|^{2}=\sup _{\|g\|=1, g \in X^{*}}\left\|L_{M}^{*} g\right\|_{\left(H^{\widehat{\otimes} n}\right)^{*}}^{2} \\
& =\sup _{\|g\|=1, g \in X^{*}} \sum_{\Delta_{n}}\left|\left\langle L_{M}^{*} g, \frac{e_{i_{1}}^{\otimes r_{1}} \widehat{\otimes} e_{i_{2}}^{\otimes r_{2}} \widehat{\otimes} \cdots \widehat{\otimes} e_{i_{k}}^{\otimes r_{k}}}{\sqrt{r_{1} ! r_{2} ! \cdots r_{k} !}}\right\rangle_{\left(H^{\widehat{\otimes} n)^{*} \times H^{\widehat{\otimes}}}\right.}\right|^{2} \\
& =\sup _{\|g\|=1, g \in X^{*}} \frac{1}{n !} \sum_{j_{1}, j_{2}, \ldots, j_{n}}\left|\left\langle L_{M}^{*} g, e_{j_{1}} \widehat{\otimes} e_{j_{2}} \widehat{\otimes} \cdots \widehat{\otimes} e_{j_{n}}\right\rangle_{\left(H^{\otimes} \widehat{\otimes}\right)^{*} \times H^{\widehat{\otimes} n}}\right|^{2} \\
& =\sup _{\|g\|=1, g \in X^{*}} \frac{1}{n !} \sum_{j_{1}, j_{2}, \ldots, j_{n}}\left|\left\langle g, L_{M}\left(e_{j_{1}} \widehat{\otimes} e_{j_{2}} \widehat{\otimes} \cdots \widehat{\otimes} e_{j_{n}}\right)\right\rangle_{X^{*} \times X}\right|^{2} \\
& =\sup _{\|g\|=1, g \in X^{*}} \frac{1}{n !} \sum_{j_{1}, j_{2}, \ldots, j_{n}}\left|\left\langle g, M\left(e_{j_{1}}, e_{j_{2}}, \ldots, e_{j_{n}}\right)\right\rangle_{X^{*} \times X}\right|^{2} \\
& =\frac{1}{n !}\|M\|_{s}^{2},
\end{aligned}
$$

where $\triangle_{n}$ denotes the relation

$$
\begin{gathered}
1 \leq i_{1}<i_{2}<\cdots<i_{k}, \quad 1 \leq r_{1}, r_{2}, \ldots, r_{k} \leq n, \\
r_{1}+r_{2}+\cdots+r_{k}=n, \quad 1 \leq k \leq n .
\end{gathered}
$$

Hence $\left\|L_{M}\right\|=\|M\|_{s} / \sqrt{n !}$.

Let $\Gamma(H)$ be the Boson Fock space over $H$, namely

$$
\Gamma(H)=\mathbb{K} \oplus H \oplus H^{\widehat{\otimes} 2} \oplus \cdots \oplus H^{\widehat{\otimes} n} \oplus \cdots .
$$

The inner product and norm of $\Gamma(H)$ are denoted by $\langle\cdot, \cdot\rangle\rangle$ and $\|\cdot\|$, respectively. It is well known that $F \in \Gamma(H)$ if and only if $F=\bigoplus_{n=0}^{\infty} u_{n}$, where $u_{n} \in H^{\otimes}, n \geq 0$ and $\sum_{n=0}^{\infty} n !\left|u_{n}\right|^{2}<\infty$. In that case, $\|F\|^{2}=\sum_{n=0}^{\infty} n !\left|u_{n}\right|^{2}$.

For each $h \in H$, let $\mathcal{E}(h)$ denote the exponential vector associated with $h$, namely

$$
\mathcal{E}(h)=1 \oplus h \oplus \frac{1}{2 !} h^{\otimes 2} \oplus \cdots \oplus \frac{1}{n !} h^{\otimes n} \oplus \cdots,
$$

which belongs to $\Gamma(H)$. Then (2.10) defines a mapping $\mathcal{E}: H \mapsto \Gamma(H)$, which is known as the exponential mapping on $H$. 
THEOREM 2.5. Let $L_{n}: H^{\widehat{\otimes} n} \mapsto X, n \geq 0$, be a sequence of bounded linear operators satisfying

$$
\sup _{\|g\|=1, g \in X^{*}} \sum_{n=0}^{\infty}\left\|L_{n}^{*} g\right\|_{\left(H^{\otimes} \widehat{n}\right)^{*}}^{2}<\infty .
$$

Then there exists a bounded linear operator $L: \Gamma(H) \mapsto X$ such that

$$
L(F)=(w) \sum_{n=0}^{\infty} L_{n} u_{n}, \quad F=\bigoplus_{n=0}^{\infty} u_{n} \in \Gamma(H)
$$

In particular,

$$
L \circ \mathcal{E}(h)=(w) \sum_{n=0}^{\infty} \frac{1}{n !} L_{n} h^{\otimes n}, \quad h \in H,
$$

where $(w)$ means weak convergence in $X$.

PROOF. Let $c=\sup _{\|g\|=1, g \in X^{*}} \sum_{n=0}^{\infty}\left\|L_{n}^{*} g\right\|_{\left(H^{\otimes}\right)^{*}}^{2}$. For $F=\bigoplus_{n=0}^{\infty} u_{n} \in \Gamma(H)$, consider the series $\sum_{n=0}^{\infty} L_{n} u_{n}$ in $X$. For each $g \in X^{*}$,

$$
\begin{aligned}
\sum_{n=0}^{\infty}\left|\left\langle g, L_{n} u_{n}\right\rangle_{X^{*} \times X}\right| & =\sum_{n=0}^{\infty}\left|\left\langle L_{n}{ }^{*} g, u_{n}\right\rangle_{\left(H^{\widehat{\otimes}}\right)^{*} \times H^{\widehat{\otimes} n}}\right| \\
& \leq\left\{\sum_{n=0}^{\infty}\left\|L_{n}{ }^{*} g\right\|_{\left(H^{\widehat{\otimes}}\right)^{*}}^{2}\right\}^{1 / 2}\left\{\sum_{n=0}^{\infty}\left\|u_{n}\right\|_{H^{\otimes} n}^{2}\right\}^{1 / 2} \leq \sqrt{c}\|g\|\|F\|,
\end{aligned}
$$

which implies that $\sum_{n=0}^{\infty} L_{n} u_{n}$ is weakly convergent in $X$. Hence there exists a unique element $F_{X^{* *}} \in X^{* *}$ such that

$$
\left\langle F_{X^{* *}}, g\right\rangle_{X^{* *} \times X^{*}}=\sum_{n=0}^{\infty}\left\langle g, L_{n} u_{n}\right\rangle_{X^{*} \times X}, \quad g \in X^{*} .
$$

Define $A(F)=F_{X^{* *}}$. Then $A: \Gamma(H) \mapsto X^{* *}$ is a linear operator and, moreover,

$$
\langle A(F), g\rangle_{X^{* *} \times X^{*}}=\sum_{n=0}^{\infty}\left\langle g, L_{n} u_{n}\right\rangle_{X^{*} \times X}, \quad F=\bigoplus_{n=0}^{\infty} u_{n} \in \Gamma(H), g \in X^{*} .
$$

For $F=\bigoplus_{n=0}^{\infty} u_{n} \in \Gamma(H)$, by (2.15), we obtain

$$
\begin{aligned}
\|A(F)\|_{X^{* *}} & =\sup _{\|g\|=1, g \in X^{*}}\left|\langle A(F), g\rangle_{X^{* *} \times X^{*}}\right| \leq \sup _{\|g\|=1, g \in X^{*}} \sum_{n=0}^{\infty}\left|\left\langle g, L_{n} u_{n}\right\rangle_{X^{*} \times X}\right| \\
& =\sup _{\|g\|=1, g \in X^{*}} \sum_{n=0}^{\infty}\left|\left\langle L_{n}^{*} g, u_{n}\right\rangle_{\left(H^{\widehat{\otimes}}\right)^{*} \times H^{\otimes} n}\right| \leq \sqrt{c}\|F\| .
\end{aligned}
$$

Therefore $A: \Gamma(H) \mapsto X^{* *}$ is a bounded linear operator. 
For each $n \geq 0$ and each $u \in H^{\widehat{\otimes} n}$, by viewing $u$ as a vector of $\Gamma(H)$ and using (2.15), we obtain

$$
\langle A u, g\rangle_{X^{* *} \times X^{*}}=\left\langle g, L_{n} u\right\rangle_{X^{*} \times X}=\left\langle J L_{n} u, g\right\rangle_{X^{* *} \times X^{*}}, \quad g \in X^{*},
$$

where $J: X \mapsto X^{* *}$ is the natural embedding mapping. This implies that

$$
A\left(H^{\widehat{\otimes} n}\right) \subset J(X), \quad n \geq 0 .
$$

Hence $A(\Gamma(H)) \subset J(X)$.

Set $L=J^{-1} A$. Then $L: \Gamma(H) \mapsto X$ is a bounded linear operator and, moreover, for $F=\bigoplus_{n=0}^{\infty} u_{n} \in \Gamma(H)$,

$$
\langle g, L(F)\rangle_{X^{*} \times X}=\langle A(F), g\rangle_{X^{* *} \times X^{*}}=\sum_{n=0}^{\infty}\left\langle g, L_{n} u_{n}\right\rangle_{X^{*} \times X}, \quad g \in X^{*},
$$

which means that

$$
L(F)=(w) \sum_{n=0}^{\infty} L_{n} u_{n}
$$

This completes the proof.

\section{Fock factorization of B-valued analytic mappings}

Throughout this section, $X$ is a complex Banach space with the norm $\|\cdot\|_{X}$. We denote by $\langle\cdot, \cdot\rangle_{X^{*} \times X}$ the canonical bilinear form on $X^{*} \times X$, where $X^{*}$ is the dual of $X$.

Let $\left\{H_{p} \mid p \in \mathbb{R}_{+}\right\}$be a given family of complex separable Hilbert spaces, $\langle\cdot, \cdot\rangle_{p}$ and $|\cdot|_{p}$ denoting the inner product and norm of $H_{p}$, respectively. We make the following fundamental assumptions: for each pair $p, q \in \mathbb{R}_{+}$with $p<q, H_{p}$ is a linear subspace of $H_{q}$ and $|\cdot|_{p}$ is stronger than $|\cdot|_{q}$ on $H_{p}$.

Definition 3.1. Let $\mathcal{N}^{*}=\bigcup_{p \in \mathbb{R}_{+}} H_{p}$ and endow $\mathcal{N}^{*}$ with the inductive limit topology corresponding to the natural embeddings of $H_{p} \mathrm{~s}$ in $\mathcal{N}^{*}$. We call $\mathcal{N}^{*}$ the Hilbert inductive limit of the family $\left\{H_{p} \mid p \in \mathbb{R}_{+}\right\}$.

For $p \in \mathbb{R}_{+}$, let $\Gamma\left(H_{p}\right)$ be the Boson Fock space over $H_{p}$ with inner product $\left\langle\langle\cdot, \cdot\rangle_{p}\right.$ and norm $\|\cdot\|_{p}$. It can be proved that for $p, q \in \mathbb{R}_{+}$with $p<q, \Gamma\left(H_{p}\right)$ is a linear subspace of $\Gamma\left(H_{q}\right)$ and, moreover, $\|\cdot\|_{p}$ is stronger than $\|\cdot\|_{q}$ on $\Gamma\left(H_{p}\right)$.

Definition 3.2. The Hilbert inductive limit of the family $\left\{\Gamma\left(H_{p}\right) \mid p \in \mathbb{R}_{+}\right\}$is called the Boson Fock space over $\mathcal{N}^{*}$ and denoted by $\Gamma\left(\mathcal{N}^{*}\right)$, namely

$$
\Gamma\left(\mathcal{N}^{*}\right)=\bigcup_{p \in \mathbb{R}_{+}} \Gamma\left(H_{p}\right)
$$

and $\Gamma\left(\mathcal{N}^{*}\right)$ is endowed with the inductive limit topology corresponding to the natural embeddings of $\Gamma\left(H_{p}\right)$ s in $\Gamma\left(\mathcal{N}^{*}\right)$. 
For each $h \in \mathcal{N}^{*}$, let $\mathcal{E}(h)$ denote the exponential vector associated with $h$, namely

$$
\mathcal{E}(h)=1 \oplus h \oplus \frac{1}{2 !} h^{\otimes 2} \oplus \cdots \oplus \frac{1}{n !} h^{\otimes n} \oplus \cdots
$$

Then (3.2) defines a mapping $\mathcal{E}: \mathcal{N}^{*} \mapsto \Gamma\left(\mathcal{N}^{*}\right)$, which is referred to as the exponential mapping on $\mathcal{N}^{*}$. Clearly, for each $p \in \mathbb{R}_{+},\left.\mathcal{E}\right|_{H_{p}}=\mathcal{E}_{p}$, where $\mathcal{E}_{p}$ denotes the exponential mapping on $H_{p}$.

The exponential mapping $\mathcal{E}: \mathcal{N}^{*} \rightarrow \Gamma\left(\mathcal{N}^{*}\right)$ is continuous and, moreover, the exponential vector set $\mathcal{E}\left(\mathcal{N}^{*}\right)$ is total in $\Gamma\left(\mathcal{N}^{*}\right)$.

Definition 3.3. A mapping $\Psi: \mathcal{N}^{*} \mapsto X$ is said to be an $X$-valued analytic mapping on $\mathcal{N}^{*}$ if the following conditions are satisfied:

(i) $\Psi$ is locally bounded on $\mathcal{N}^{*}$;

(ii) for each $f, h \in \mathcal{N}^{*}$ and $g \in X^{*}$, the complex function

$$
z: \mapsto\langle g, \Psi(f+z h)\rangle_{X^{*} \times X}
$$

is an entire function on $\mathbb{C}$.

REMARK 3.4. Let $\Psi: \mathcal{N}^{*} \mapsto X$ be an $X$-valued analytic mapping on $\mathcal{N}^{*}$. Then, by the Graves-Taylor-Hille-Zorn theorem (see, for example, [1]), for each $p \in \mathbb{R}_{+}$, the restriction $\Psi_{p}=\left.\Psi\right|_{H_{p}}$ is an $X$-valued analytic mapping on the Hilbert space $H_{p}$. Hence, for each $p \in \mathbb{R}_{+}, \Psi_{p}=\left.\Psi\right|_{H_{p}}$ is infinitely Fréchet differentiable on $H_{p}$ and, moreover, for each $f \in H_{p}$ there exists a constant $r>0$ such that

$$
\Psi_{p}(h)=\sum_{n=0}^{\infty} \frac{1}{n !} \Psi_{p}^{(n)}(f)(h-f)^{n}, \quad h \in B_{H_{p}}(f, r),
$$

where $B_{H_{p}}(f, r)=\left\{h \in H_{p}|| h-\left.f\right|_{p}<r\right\}$ and the series is convergent in the norm of $X$.

From this remark, we easily arrive at the next proposition.

Proposition 3.5. Let $\Psi: \mathcal{N}^{*} \mapsto X$ be an $X$-valued analytic mapping on $\mathcal{N}^{*}$. Then for each $n \geq 1$ and each $f \in \mathcal{N}^{*}$, there exists a continuous symmetric $n$-linear mapping $\Psi^{(\bar{n})}(f): \mathcal{N}^{* n} \mapsto X$ such that

$$
\left.\Psi^{(n)}(f)\right|_{H_{p}^{n}}=\Psi_{p}^{(n)}(f), \quad p \in \mathbb{R}_{+} \text {and } f \in H_{p},
$$

where $\Psi_{p}=\left.\Psi\right|_{H_{p}}$.

THEOREM 3.6. Let $T: \Gamma\left(\mathcal{N}^{*}\right) \mapsto X$ be a continuous linear operator and $\Psi=T \circ \mathcal{E}$. Then $\Psi$ is an $X$-valued analytic mapping on $\mathcal{N}^{*}$ and, moreover, for each $p \in \mathbb{R}_{+}$,

$$
\|\Psi\|_{p}^{2} \equiv \sup _{\|g\|=1, g \in X^{*}} \sum_{n=0}^{\infty} \frac{1}{n !} \sum_{j_{1}, j_{2}, \ldots, j_{n}}\left|\left\langle g, \Psi^{(n)}(0)\left(e_{j_{1}}, e_{j_{2}}, \ldots, e_{j_{n}}\right)\right\rangle_{X^{*} \times X}\right|^{2}<\infty,
$$

where $\left\{e_{k}\right\}_{k \geq 1}$ is an orthonormal basis of $H_{p}$. 
Proof. It is easy to check that $\Psi$ is an $X$-valued analytic mapping on $\mathcal{N}^{*}$.

Let $p \in \mathbb{R}_{+}$. Set $M_{0}=T 1$ and define $M_{n}: H_{p}^{n} \mapsto X$ for $n \geq 1$ as follows:

$$
M_{n}\left(h_{1}, h_{2}, \ldots, h_{n}\right)=T_{p}\left(h_{1} \widehat{\otimes} h_{2} \widehat{\otimes} \cdots \widehat{\otimes} h_{n}\right), \quad\left(h_{1}, h_{2}, \ldots, h_{n}\right) \in H_{p}^{n},
$$

where $T_{p}=\left.T\right|_{\Gamma\left(H_{p}\right)}$, which is a bounded linear operator from $\Gamma\left(H_{p}\right)$ to $X$.

Clearly, for each $n \geq 1, M_{n}: H_{p}^{n} \mapsto X$ is a bounded symmetric $n$-linear mapping and, moreover,

$$
\left\|M_{n}\right\| \leq \sqrt{n !}\left\|T_{p}\right\|
$$

which implies $\left[(1 / n !)\left\|M_{n}\right\|\right]^{1 / n} \rightarrow 0(n \rightarrow \infty)$. On the other hand, by a simple computation, we get

$$
\Psi(h)=\Psi_{p}(h)=T_{p} \circ \mathcal{E}_{p}(h)=\sum_{n=0}^{\infty} \frac{1}{n !} M_{n} h^{n}, \quad h \in H_{p},
$$

where $\Psi_{p}=\left.\Psi\right|_{H_{p}}$ and $T_{p}=\left.T\right|_{\Gamma\left(H_{p}\right)}$. This, together with Proposition 3.5, yields that

$$
\Psi^{(n)}(0)=\Psi_{p}^{(n)}(0)=M_{n}, \quad n \geq 0 .
$$

Let $\left\{e_{k}\right\}_{k \geq 1}$ be an orthonormal basis of $H_{p}$. Then

$$
\begin{aligned}
\|\Psi\|_{p}^{2} & \equiv \sup _{\|g\|=1, g \in X^{*}} \sum_{n=0}^{\infty} \frac{1}{n !} \sum_{j_{1}, j_{2}, \ldots, j_{n}}\left|\left\langle g, \Psi^{(n)}(0)\left(e_{j_{1}}, e_{j_{2}}, \ldots, e_{j_{n}}\right)\right\rangle_{X^{*} \times X}\right|^{2} \\
& =\sup _{\|g\|=1, g \in X^{*}} \sum_{n=0}^{\infty} \frac{1}{n !} \sum_{j_{1}, j_{2}, \ldots, j_{n}}\left|\left\langle g, M_{n}\left(e_{j_{1}}, e_{j_{2}}, \ldots, e_{j_{n}}\right)\right\rangle_{X^{*} \times X}\right|^{2} \\
& =\sup _{\|g\|=1, g \in X^{*}} \sum_{n=0}^{\infty} \frac{1}{n !} \sum_{j_{1}, j_{2}, \ldots, j_{n}}\left|\left\langle g, T_{p}\left(e_{j_{1}} \widehat{\otimes} e_{j_{2}} \widehat{\otimes} \cdots \widehat{\otimes} e_{j_{n}}\right)\right\rangle_{X^{*} \times X}\right|^{2} .
\end{aligned}
$$

On the other hand,

$$
\begin{aligned}
\left\|T_{p}\right\|^{2} & =\left\|T_{p}^{*}\right\|^{2}=\sup _{\|g\|=1, g \in X^{*}}\left\|T_{p}^{*} g\right\|_{\Gamma\left(H_{p}\right)^{*}}^{2} \\
& =\sup _{\|g\|=1, g \in X^{*}} \sum_{n=0}^{\infty} \sum_{\Delta_{n}}\left|\left\langle T_{p}^{*} g, \frac{e_{i_{1}}^{\otimes r_{1}} \widehat{\otimes} e_{i_{2}}^{\otimes r_{2}} \widehat{\otimes} \cdots \widehat{\otimes} e_{i_{k}}^{\otimes r_{k}}}{\sqrt{r_{1} ! r_{2} ! \cdots r_{k} !}}\right\rangle_{\Gamma\left(H_{p}\right)^{*} \times \Gamma\left(H_{p}\right)}\right|^{2} \\
& =\sup _{\|g\|=1, g \in X^{*}} \sum_{n=0}^{\infty} \sum_{\Delta_{n}} \mid\left\langle g,\left.\left.T_{p}\left(\frac{e_{i_{1}}^{\otimes r_{1}} \widehat{\otimes} e_{i_{2}}^{\otimes r_{2}} \widehat{\otimes} \cdots \widehat{\otimes} e_{i_{k}}^{\otimes r_{k}}}{\sqrt{r_{1} ! r_{2} ! \cdots r_{k} !}}\right)\right|_{X^{*} \times X}\right|^{2}\right. \\
& =\sup _{\|g\|=1, g \in X^{*}} \sum_{n=0}^{\infty} \frac{1}{n !} \sum_{j_{1}, j_{2}, \ldots, j_{n}}\left|\left\langle g, T_{p}\left(e_{j_{1}} \widehat{\otimes} e_{j_{2}} \widehat{\otimes} \cdots \widehat{\otimes} e_{j_{n}}\right)\right\rangle_{X^{*} \times X}\right|^{2},
\end{aligned}
$$

where $\triangle_{n}$ is defined by (2.8). Therefore $\|\Psi\|_{p}^{2}=\left\|T_{p}\right\|^{2}<\infty$. 
Lemma 3.7. Let $\Psi: \mathcal{N}^{*} \mapsto X$ be an $X$-valued analytic mapping on $\mathcal{N}^{*}$. Assume that, for each $p \in \mathbb{R}_{+}$,

$$
\|\Psi\|_{p}^{2} \equiv \sup _{\|g\|=1, g \in X^{*}} \sum_{n=0}^{\infty} \frac{1}{n !} \sum_{j_{1}, j_{2}, \ldots, j_{n}}\left|\left\langle g, \Psi^{(n)}(0)\left(e_{j_{1}}, e_{j_{2}}, \ldots, e_{j_{n}}\right)\right\rangle_{X^{*} \times X}\right|^{2}<\infty
$$

where $\left\{e_{k}\right\}_{k \geq 1}$ is an orthonormal basis of $H_{p}$. Then, for each $p \in \mathbb{R}_{+}$, there exists a bounded linear operator $T_{p}: \Gamma\left(H_{p}\right) \mapsto X$ such that

$$
\Psi_{p}=T_{p} \circ \mathcal{E}_{p}
$$

where $\Psi_{p}=\left.\Psi\right|_{H_{p}}$ and $\mathcal{E}_{p}$ is the exponential mapping on $H_{p}$.

PROOF. Let $p \in \mathbb{R}_{+}$. By Remark 3.4, there exists a constant $r>0$ such that

$$
\Psi_{p}(h)=\sum_{n=0}^{\infty} \frac{1}{n !} \Psi_{p}^{(n)}(0) h^{n}, \quad h \in B_{H_{p}}(0, r),
$$

where $B_{H_{p}}(0, r)=\left\{\left.h \in H_{p}|| h\right|_{p}<r\right\}$ and the series is convergent in the norm of $X$.

For the orthonormal basis $\left\{e_{k}\right\}_{k \geq 1}$ of $H_{p}$, by Proposition 3.5,

$$
\begin{aligned}
\frac{1}{n !}\left\|\Psi_{p}^{(n)}(0)\right\|_{s}^{2} & =\sup _{\|g\|=1, g \in X^{*}} \frac{1}{n !} \sum_{j_{1}, j_{2}, \ldots, j_{n}}\left|\left\langle g, \Psi_{p}^{(n)}(0)\left(e_{j_{1}}, e_{j_{2}}, \ldots, e_{j_{n}}\right)\right\rangle_{X^{*} \times X}\right|^{2} \\
& \leq \sup _{\|g\|=1, g \in X^{*}} \sum_{n=0}^{\infty} \frac{1}{n !} \sum_{j_{1}, j_{2}, \ldots, j_{n}}\left|\left\langle g, \Psi_{p}^{(n)}(0)\left(e_{j_{1}}, e_{j_{2}}, \ldots, e_{j_{n}}\right)\right\rangle_{X^{*} \times X}\right|^{2} \\
& =\sup _{\|g\|=1, g \in X^{*}} \sum_{n=0}^{\infty} \frac{1}{n !} \sum_{j_{1}, j_{2}, \ldots, j_{n}}\left|\left\langle g, \Psi^{(n)}(0)\left(e_{j_{1}}, e_{j_{2}}, \ldots, e_{j_{n}}\right)\right\rangle_{X^{*} \times X}\right|^{2} \\
& =\|\Psi\|_{p}^{2}<\infty .
\end{aligned}
$$

This shows that $\Psi_{p}^{(n)}(0): H_{p}^{n} \mapsto X$ is strongly bounded for each $n \geq 0$ and, moreover,

$$
\frac{1}{n !}\left\|\Psi_{p}^{(n)}(0)\right\| \leq \frac{1}{n !}\left\|\Psi_{p}^{(n)}(0)\right\|_{s} \leq \frac{1}{\sqrt{n !}}\|\Psi\|_{p}, \quad n \geq 0 .
$$

Hence

$$
\lim _{n \rightarrow \infty}\left[\frac{1}{n !}\left\|\Psi_{p}^{(n)}(0)\right\|\right]^{1 / n}=0
$$

which implies that the series $\sum_{n=0}^{\infty}(1 / n !) \Psi_{p}^{(n)}(0) h^{n}$ is absolutely convergent in $X$ for each $h \in H_{p}$.

On the other hand, the complex function

$$
z: \mapsto\left\langle g, \Psi_{p}(z h)\right\rangle_{X^{*} \times X}=\langle g, \Psi(z h)\rangle_{X^{*} \times X}
$$


is an entire function on $\mathbb{C}$ for each pair $g \in X^{*}$ and $h \in H_{p}$. Hence

$$
\begin{aligned}
\left\langle g, \Psi_{p}(h)\right\rangle_{X^{*} \times X} & =\left.\sum_{n=0}^{\infty} \frac{1}{n !} \frac{d^{n}}{d z^{n}}\left\langle g, \Psi_{p}(z h)\right\rangle_{X^{*} \times X}\right|_{z=0}=\sum_{n=0}^{\infty} \frac{1}{n !}\left\langle g, \Psi_{p}^{(n)}(0) h^{n}\right\rangle_{X^{*} \times X} \\
& =\left\langle g, \sum_{n=0}^{\infty} \frac{1}{n !} \Psi_{p}^{(n)}(0) h^{n}\right\rangle_{X^{*} \times X}, \quad g \in X^{*}, h \in H_{p},
\end{aligned}
$$

which shows that

$$
\Psi_{p}(h)=\sum_{n=0}^{\infty} \frac{1}{n !} \Psi_{p}^{(n)}(0) h^{n}, \quad h \in H_{p} .
$$

We now take another look at $\Psi_{p}^{(n)}(0): H_{p}^{n} \mapsto X, n \geq 1$, which are symmetric and strongly bounded as is shown above. By Theorem 2.4, there exist bounded linear operators $L_{n}: H_{p}^{\widehat{\otimes} n} \mapsto X, n \geq 1$, such that $\left\|L_{n}\right\|=\left\|\Psi_{p}^{(n)}(0)\right\|_{s} / \sqrt{n !}$ and

$$
\Psi_{p}^{(n)}(0)\left(h_{1}, h_{2}, \ldots, h_{n}\right)=L_{n}\left(h_{1} \widehat{\otimes} h_{2} \widehat{\otimes} \ldots \widehat{\otimes} h_{n}\right), \quad\left(h_{1}, h_{2}, \ldots, h_{n}\right) \in H_{p}^{n}, n \geq 1 .
$$

Let

$$
L_{0} z=z \Psi_{p}^{(0)}(0)=z \Psi_{p}(0), \quad z \in H_{p}^{\widehat{\otimes} 0} \equiv \mathbb{C}
$$

Then

$$
\begin{aligned}
\sup _{\|g\|=1, g \in X^{*}} & \sum_{n=0}^{\infty} \|\left. L_{n}^{*} g\right|_{\left(H_{p}^{\widehat{\otimes} n}\right)^{*}} ^{2} \\
= & \sup _{\|g\|=1, g \in X^{*}} \sum_{n=0}^{\infty} \sum_{\Delta_{n}}\left|\left\langle L_{n}^{*} g, \frac{e_{i_{1}}^{\otimes r_{1}} \widehat{\otimes} e_{i_{2}}^{\otimes r_{2}} \widehat{\otimes} \cdots \widehat{\otimes} e_{i_{k}}^{\otimes r_{k}}}{\sqrt{r_{1} ! r_{2} ! \cdots r_{k} !}}\right\rangle_{\left(H_{p}^{\otimes n}\right)^{*} \times H_{p}^{\otimes n}}\right|^{2} \\
= & \sup _{\|g\|=1, g \in X^{*}} \sum_{n=0}^{\infty} \sum_{\triangle_{n}} \mid\left\langle g,\left.\left.L_{n}\left(\frac{e_{i_{1}}^{\otimes r_{1}} \widehat{\otimes} e_{i_{2}}^{\otimes r_{2}} \widehat{\otimes} \cdots \widehat{\otimes} e_{i_{k}}^{\otimes r_{k}}}{\sqrt{r_{1} ! r_{2} ! \cdots r_{k} !}}\right)\right|_{X^{*} \times X}\right|^{2}\right. \\
= & \sup _{\|g\|=1, g \in X^{*}} \sum_{n=0}^{\infty} \frac{1}{n !} \sum_{j_{1}, j_{2}, \ldots, j_{n}}\left|\left\langle g, L_{n}\left(e_{j_{1}} \widehat{\otimes} e_{j_{2}} \widehat{\otimes} \cdots \widehat{\otimes} e_{j_{n}}\right)\right\rangle_{X^{*} \times X}\right|^{2} \\
= & \sup _{\|g\|=1, g \in X^{*}} \sum_{n=0}^{\infty} \frac{1}{n !} \sum_{j_{1}, j_{2}, \ldots, j_{n}}\left|\left\langle g, \Psi_{p}^{(n)}(0)\left(e_{j_{1}}, e_{j_{2}}, \ldots, e_{j_{n}}\right)\right\rangle_{X^{*} \times X}\right|^{2} \\
= & \sup _{\|g\|=1, g \in X^{*}} \sum_{n=0}^{\infty} \frac{1}{n !} \sum_{j_{1}, j_{2}, \ldots, j_{n}}\left|\left\langle g, \Psi^{(n)}(0)\left(e_{j_{1}}, e_{j_{2}}, \ldots, e_{j_{n}}\right)\right\rangle_{X^{*} \times X}\right|^{2} \\
= & \|\Psi\|_{p}^{2}<\infty,
\end{aligned}
$$

where $\left\{e_{k}\right\}_{k \geq 1}$ is the orthonormal basis of $H_{p}$ as is shown in the conditions. Therefore, 
by Theorem 2.5 , there exists a bounded linear operator $T_{p}: \Gamma\left(H_{p}\right) \mapsto X$ such that

$$
T_{p} \circ \mathcal{E}_{p}(h)=(w) \sum_{n=0}^{\infty} \frac{1}{n !} L_{n} h^{\otimes n}, \quad h \in H_{p},
$$

where $(w)$ means weak convergence in $X$. This, together with (3.10) and (3.11), yields that

$$
\Psi_{p}(h)=T_{p} \circ \mathcal{E}_{p}(h), \quad h \in H_{p} .
$$

This completes the proof.

Lemma 3.8. Let $\Psi: \mathcal{N}^{*} \mapsto X$ be an $X$-valued analytic mapping on $\mathcal{N}^{*}$. Assume that, for each $p \in \mathbb{R}_{+}$, there exists a bounded linear operator $T_{p}: \Gamma\left(H_{p}\right) \mapsto X$ such that

$$
\Psi_{p}=T_{p} \circ \mathcal{E}_{p}
$$

where $\Psi_{p}=\left.\Psi\right|_{H_{p}}$ and $\mathcal{E}_{p}$ is the exponential mapping on $H_{p}$. Then there exists a continuous linear operator $T: \Gamma\left(\mathcal{N}^{*}\right) \mapsto X$ such that $\Psi=T \circ \mathcal{E}$.

PROOF. Consider the operator family $\left\{T_{p}\right\}_{p \in \mathbb{R}_{+}}$. For each pair $p, q \in \mathbb{R}_{+}$with $p \leq q$, we assert that

$$
T_{p} u=T_{q} u, \quad u \in \Gamma\left(H_{p}\right) .
$$

In fact, the restriction $\left.T_{q}\right|_{\Gamma\left(H_{p}\right)}$ is bounded with respect to norm $\|\cdot\|_{p}$ since $\|\cdot\|_{p}$ is stronger than $\|\cdot\|_{q}$ on $\Gamma\left(H_{p}\right)$. On the other hand, for each $h \in H_{p}$,

$$
T_{p}\left(\mathcal{E}_{p}(h)\right)=\Psi_{p}(h)=\Psi_{q}(h)=T_{q}\left(\mathcal{E}_{q}(h)\right)=\left.T_{q}\right|_{\Gamma\left(H_{p}\right)}\left(\mathcal{E}_{p}(h)\right),
$$

which implies that $T_{p}=\left.T_{q}\right|_{\Gamma\left(H_{p}\right)}$ since $\left\{\mathcal{E}_{p}(h) \mid h \in H_{p}\right\}$ is total in $\Gamma\left(H_{p}\right)$.

Now define a mapping $T: \Gamma\left(\mathcal{N}^{*}\right) \mapsto X$ as follows. For each $u \in \Gamma\left(\mathcal{N}^{*}\right)$, put

$$
T u=T_{p} u \quad \text { if } u \in \Gamma\left(H_{p}\right) .
$$

It is easy to see that $T: \Gamma\left(\mathcal{N}^{*}\right) \mapsto X$ is a well-defined linear operator and, moreover, for each $p \in \mathbb{R}_{+}$, the restriction of $T$ on $\Gamma\left(H_{p}\right)$ is exactly $T_{p}$, which is continuous. It then follows that $T: \Gamma\left(\mathcal{N}^{*}\right) \mapsto X$ is continuous.

Finally, we show that $\Psi=T \circ \mathcal{E}$. Indeed, for each $h \in \mathcal{N}^{*}$, there exists a $p \in \mathbb{R}_{+}$ such that $h \in H_{p}$, hence

$$
\Psi(h)=\left.\Psi\right|_{p}(h)=T_{p}\left(\mathcal{E}_{p}(h)\right)=T(\mathcal{E}(h))=T \circ \mathcal{E}(h) .
$$

This completes the proof.

Lemmas 3.7 and 3.8 easily lead to the next theorem. 
Theorem 3.9. Let $\Psi: \mathcal{N}^{*} \mapsto X$ be an $X$-valued analytic mapping on $\mathcal{N}^{*}$. Assume that, for each $p \in \mathbb{R}_{+}$,

$$
\|\Psi\|_{p}^{2} \equiv \sup _{\|g\|=1, g \in X^{*}} \sum_{n=0}^{\infty} \frac{1}{n !} \sum_{j_{1}, j_{2}, \ldots, j_{n}}\left|\left\langle g, \Psi^{(n)}(0)\left(e_{j_{1}}, e_{j_{2}}, \ldots, e_{j_{n}}\right)\right\rangle_{X^{*} \times X}\right|^{2}<\infty
$$

where $\left\{e_{k}\right\}_{k \geq 1}$ is an orthonormal basis of $H_{p}$. Then there exists a continuous linear operator $T: \Gamma\left(\mathcal{N}^{*}\right) \mapsto X$ such that $\Psi=T \circ \mathcal{E}$.

The next corollary, an immediate consequence of Theorems 3.6 and 3.9, provides a necessary and sufficient condition for an $X$-valued analytic mapping $\Psi$ on $\mathcal{N}^{*}$ to have a factorization of the form $\Psi=T \circ \mathcal{E}$.

Corollary 3.10. Let $\Psi: \mathcal{N}^{*} \mapsto X$ be an $X$-valued analytic mapping on $\mathcal{N}^{*}$. Then the following two conditions are equivalent:

(i) there exists a continuous linear operator $T: \Gamma\left(\mathcal{N}^{*}\right) \mapsto X$ such that $\Psi=T \circ \mathcal{E}$;

(ii) for each $p \in \mathbb{R}_{+}$,

$$
\|\Psi\|_{p}^{2} \equiv \sup _{\|g\|=1, g \in X^{*}} \sum_{n=0}^{\infty} \frac{1}{n !} \sum_{j_{1}, j_{2}, \ldots, j_{n}}\left|\left\langle g, \Psi^{(n)}(0)\left(e_{j_{1}}, e_{j_{2}}, \ldots, e_{j_{n}}\right)\right\rangle_{X^{*} \times X}\right|^{2}<\infty,
$$

where $\left\{e_{k}\right\}_{k \geq 1}$ is an orthonormal basis of $H_{p}$.

\section{Application}

In this final section, we show an application of our main results to white noise testing functionals.

Let $(E) \subset\left(L^{2}\right) \subset(E)^{*}$ be the classical white noise analysis framework over a real Gel'fand triple

$$
E \subset \mathcal{H} \subset E^{*}
$$

Elements of $(E)$ are known as white noise testing functionals, while elements of $(E)^{*}$ are known as white noise generalized functionals (see, for example, [2, 3]).

It is known (see, for example, [2,3]) that there exists a family $\left\{E_{-p} \mid p \in \mathbb{R}_{+}\right\}$of real separable Hilbert spaces satisfying the following conditions:

(i) for each pair $p, q \in \mathbb{R}_{+}$with $p<q, E_{-p}$ is a linear subspace of $E_{-q}$ and $|\cdot|_{-p}$ is stronger than $|\cdot|_{-q}$ on $E_{-p}$, where $|\cdot|_{-p}$ means the norm of $E_{-p}$;

(ii) $E^{*}$ is the Hilbert inductive limit of the family $\left\{E_{-p} \mid p \in \mathbb{R}_{+}\right\}$, namely

$$
E^{*}=\bigcup_{p \in \mathbb{R}_{+}} E_{-p}
$$

and $E^{*}$ is endowed with the inductive limit topology. 
For each $p \in \mathbb{R}_{+}$, put $H_{p}=E_{-p, c}$, the complexification of $E_{-p}$, which is a complex separable Hilbert space. We denote by $\langle\cdot, \cdot\rangle_{p}$ and $|\cdot|_{p}$ the inner product and norm of $H_{p}$, respectively. It is easy to see that for each pair $p, q \in \mathbb{R}_{+}$with $p<q$, $H_{p}$ is a linear subspace of $H_{q}$ and $|\cdot|_{p}$ is stronger than $|\cdot|_{q}$ on $H_{p}$.

Let $\mathcal{N}^{*}$ be the Hilbert inductive limit of the family $\left\{H_{p} \mid p \in \mathbb{R}_{+}\right\}$, namely that $\mathcal{N}^{*}=\bigcup_{p \in \mathbb{R}_{+}} H_{p}$ and $\mathcal{N}^{*}$ is endowed with the inductive limit topology corresponding to the natural embeddings of $H_{p}$ s in $\mathcal{N}^{*}$. Let $\Gamma\left(\mathcal{N}^{*}\right)$ be the Boson Fock space over $\mathcal{N}^{*}$ and $\mathcal{E}$ the exponential mapping on $\mathcal{N}^{*}$ (see Section 3 for the definitions).

Through the Wiener-Itô-Segal isomorphism (see, for example, [2, 3]), we can identify $(E)^{*}$ with $\Gamma\left(\mathcal{N}^{*}\right)$. Hence each white noise testing functional $\varphi \in(E)$ can be viewed as a $\mathbb{C}$-valued continuous linear functional on $\Gamma\left(\mathcal{N}^{*}\right)$ and the composition $\varphi \circ \mathcal{E}$ then makes sense. Moreover, by Theorem 3.6, $\varphi \circ \mathcal{E}$ is a $\mathbb{C}$-valued analytic functional on $\mathcal{N}^{*}$, where $\mathcal{E}$ is the exponential mapping on $\mathcal{N}^{*}$.

Applying Corollary 3.10, we come to the next characterization theorem for white noise testing functionals.

THEOREM 4.1. Let $\Psi: \mathcal{N}^{*} \mapsto \mathbb{C}$ be a $\mathbb{C}$-valued analytic functional on $\mathcal{N}^{*}$. Then the following two conditions are equivalent:

(i) there exists a white noise testing functional $\varphi \in(E)$ such that $\Psi=\varphi \circ \mathcal{E}$;

(ii) for each $p \in \mathbb{R}_{+}$,

$$
\|\Psi\|_{p}^{2} \equiv \sum_{n=0}^{\infty} \frac{1}{n !} \sum_{j_{1}, j_{2}, \ldots, j_{n}}\left|\Psi^{(n)}(0)\left(e_{j_{1}}, e_{j_{2}}, \ldots, e_{j_{n}}\right)\right|^{2}<\infty,
$$

where $\left\{e_{k}\right\}_{k \geq 1}$ is an orthonormal basis of $H_{p}$.

Proof. Taking $X=\mathbb{C}$ in Corollary 3.10, we find that (3.17) becomes (4.2). On the other hand, as is shown above, each white noise testing functional $\varphi \in(E)$ can be viewed as a continuous linear operator $\varphi: \Gamma\left(\mathcal{N}^{*}\right) \mapsto \mathbb{C}$. Hence, by Corollary 3.10, we conclude the proof.

\section{References}

[1] S. B. Chae, Holomorphy and Calculus in Normed Spaces (Marcel Dekker, New York, 1985).

[2] T. Hida, H.-H. Kuo, J. Potthoff and L. Streit, White Noise-An Infinite Dimensional Calculus (Kluwer Academic, Dordrecht, 1993).

[3] Z. Y. Huang and J. A. Yan, Introduction to Infinite Dimensional Stochastic Analysis (Science Press/Kluwer Acdemic, Beijing/Dordrecht, 1997).

[4] H.-H. Kuo, White Noise Distribution Theory (CRC Press, Boca Raton, FL, 1996).

[5] N. Obata, White Noise Calculus and Fock Space, Lecture Notes in Mathematics, 1577 (Springer, Berlin, 1994).

CAISHI WANG, School of Mathematics and Information Science, Northwest Normal University, Lanzhou, Gansu 730070, PR China e-mail: wangcs@nwnu.edu.cn, cswangnwnu@163.com 
YULAN ZHOU, School of Mathematics and Information Science, Northwest Normal University, Lanzhou, Gansu 730070, PR China e-mail: zhouylw@nwnu.edu.cn

DECHENG FENG, School of Mathematics and Information Science, Northwest Normal University, Lanzhou, Gansu 730070, PR China e-mail: fengdc@nwnu.edu.cn

QI HAN, School of Mathematics and Information Science, Northwest Normal University, Lanzhou, Gansu 730070, PR China e-mail: hanqi1978@nwnu.edu.cn 